\title{
CONTINUOUS CURVES IN WHICH EVERY ARC MAY BE EXTENDED*
}

\author{
BY W. L. AYRES $\dagger$
}

1. Introduction. An $\operatorname{arc} \alpha$ belonging to a point set $K$ is said to be a maximal arc of $K$ if there is no arc which belongs to $K$ and contains the arc $\alpha$ as a proper subset. S. Mazurkiewicz $\ddagger$ proved in 1921 that every arc of a bounded acyclic continuous curve $M$ is a subset of a maximal arc of $M$. However there are many types of continuous curves in which every arc of a continuous curve is a subset of a maximal arc and which are not acyclic; and in a recent paper\| we have given a necessary and sufficient condition in order that a continuous curve have this property. In this note we shall treat the opposite problem of finding under what conditions no arc is a subset of a maximal arc. We shall say that an arc $\alpha$ of a point set $K$ is extendible in $K$ if there exists an $\operatorname{arc} \beta$ of $K$ of which $\alpha$ is a proper subset. If $X$ and $Y$ are the end points of $\alpha$ and $Y$ is an interior point of $\beta$, then we say that $\alpha$ is extendible in $K$ in the direction $X Y$. And if there exists an $\operatorname{arc} \beta$ of $K$ containing $\alpha$ and such that both end points of $\alpha$ are interior points of $\beta$, then we say $\alpha$ is extendible in $K$ in both directions. In this note we shall give two necessary and suffi-

* Presented to the Society, December 27, 1928.

$\dagger$ National Research Fellow in Mathematics.

$\ddagger$ Un théorème sur les lignes de Jordan, Fundamenta Mathematicae, vol. 2 (1921), pp. 119-130. See Lemma 13, p. 129.

$\S$ A continuous curve is a closed connected, and connected im kleinen point set. A continuous curve is acyclic if it contains no simple closed curve. It must be noticed that under these definitions a single point is a continuous curve and is acyclic. However, to avoid trivial cases, we will assume that the continuous curve $M$, which is mentioned repeatedly, contains more than one point.

$\|$ W. L. Ayres, Conditions under which every arc of a continuous curve is a subset of a maximal arc of the curve, Mathematische Annalen, vol.101 (1929), pp. 194-209. 
cient conditions that every arc of a continuous curve $M$ be extendible in $M$ in both directions. The first of these conditions is that $M$ shall not contain a certain type of point. From this it may be seen that in order that every arc of $M$ be merely extendible in $M$ it is necessary and sufficient that $M$ contain at most one point of this type.

All of the point sets mentioned in this paper are assumed to lie in a two-dimensional euclidean space $E_{2}$.

2. Preliminary Lemmas. P. Urysohn* has shown that every point of a continuous curve may be $\epsilon$-separated, $\dagger$ for any positive number $\epsilon$, by a set consisting of a finite number of continua. By a result due to $G$. T. Whyburn and the author, $\ddagger$ each of these continua may be replaced by a continuous curve so that we still have an $\epsilon$-separation. Hence in characterizing types of points of a continuous curve through their $\epsilon$-separating sets we may consider only the sets of continuous curves which form an $\epsilon$-separating set. In the plane we may show that we need consider only arcs $\S$ and simple closed curves.

Lemma A. If $P$ is a point of a continuous curve $M$ and $\epsilon$ is a positive number, there exists a simple closed curve $J$ of

* Ueber im kleinen zusammenhängende Kontinua, Mathematische Annalen, vol. 98 (1927), p. 297.

$\dagger$ A set $B$ is said to be an $\epsilon$-separation of the point $P$ in the set $M$ if $M-B^{\prime}=A+C$, where $A$ and $C$ are mutually separated sets and $A$ contains the point $P$, and further the diameter of the set $A+B$ is less than $\epsilon$. See P. Urysohn, Mémoire sur les multiplicités Cantoriennes, Fundamenta Mathematicae, vol. 7 (1925), p. 65.

$\ddagger$ On continuous curves in $n$ dimensions, this Bulletin, vol. 34 (1928), pp. 349-360, Theorem 1. For two quite similar theorems published at approximately the same time, see P. Alexandroff and L. Tumarkin, Beweis des Satzes, dass jede abgeschlossene Menge positiver Dimension in einem lokal zusammenhängenden Kontinuum von derselben Dimension topologisch enthalten ist, Fundamenta Mathematicae, vol. 11 (1928), pp. 141-144; and W. Stepanoff and L. Tumarkin, Ueber eine Erweiterung abgeschlossener Mengen zu Jordanschen Kontinuen derselben Dimension, Fundamenta Mathematicae, vol. 12 (1928), pp. 43-46. However for the present application we require the property (2) of the theorem by Whyburn and myself. This property is stated in neither of the latter two theorems.

$\S$ We consider a point as a special case of an arc. 
diameter less than $\epsilon$, enclosing $P$ and such that $J \cdot M$ consists of a finite number of components, that is, every point of $M$ may be $\epsilon$-separated by a simple closed curve or a finite set of mutually exclusive arcs. If there exists a positive number $r$ and a positive integer $n$ such that, for every circle $C$ with center $P$ and radius less than or equal to $r$, the point $P$ belongs to the boundary of exactly $n(n>0)$ complementary domains of $M>C$, then $P$ may be $\epsilon$-separated in $M$ by a set of $n$ arcs, for any positive number $\epsilon$.

If $P$ belongs to the boundary of no complementary domain of $M$, then $M$ contains a simple closed curve which encloses $P$ and is of diameter less than $\epsilon^{*}$. If $P$ belongs to the boundary of a complementary domain $D$ of $M$, let $C$ be a circle with center $P$ and radius less than $\epsilon / 2$ and such that $C$ contains a point of $D$. Now using a slight modification of the proof of Theorem 1 of our paper, On the separation of points of a continuous curve by arcs and simple closed curves, $\dagger$ we may complete our proof.

Lemma B. If the point $P$ of the continuous curve $M$ may be $\epsilon$-separated, for any positive number $\epsilon$, by a set $S$ of $n$ mutually exclusive continua such that $P$ is not contained in a bounded complementary domain of any one, but not by any set $S$ of less than $n$, then $P$ may be $\epsilon$-separated by a set of $n$ mutually exclusive arcs.

For each positive integer $k$, let $C_{k}$ be a circle with center $P$ and radius $1 / k$ and let $\pi_{k}$ be the number of complementary domains of $M+C_{k}$ that have $P$ and a point of $C_{k}$ on their boundaries. Evidently $\pi_{k+1} \geqq \pi_{k}$ for every $k$. If the number $\pi_{k}$ increases indefinitely with $k$, then it is easy to see that the number of components of any $\epsilon$-separating set must increase indefinitely as $\epsilon$ approaches zero. But by hypothesis, $n$ components suffice. Hence there is an integer $s$ such that for $t \geqq s, \pi_{t}=\pi_{s}$. First let us suppose that $\pi_{s}>0$.

* G. T. Whyburn, Concerning continua in the plane, Transactions of this Society, vol. 29 (1927), p. 381, Theorem 7.

$\dagger$ Proceedings of the National Academy of Sciences, vol. 14 (1928), pp. 201-206. 
It follows from Lemma $A$ that $P$ may be $\epsilon$-separated by a set of $\pi_{s}$ mutually exclusive arcs for any $\epsilon>0$. By our hypothesis, $\pi_{s} \geqq n$. And it is easy to see that for $\epsilon<1 / s$, no $\epsilon$-separating set has less than $\pi_{s}$ components. But $P$ may be $\epsilon$-separated by a set of $n$ continua for each $\epsilon$. Therefore $\pi_{s} \leqq n$. Hence $\pi_{s}=n$ and our lemma is proved for the case $\pi_{s}>0$.

Now consider $\pi_{s}=0$. There are two cases according as $P$ is or is not a limit point of $E_{2}-M$. If $P$ is not a limit point of $E_{2}-M$, there exists a positive number $r$ such that every point of the interior of a circle with center $P$ and radius $r$ belongs to $M$. Then for $\epsilon<r / 2$ there is no $\epsilon$-separating set $S$ such that $P$ is not contained in a bounded complementary domain of some one component of $S$. Hence this case is impossible. In the remaining case we shall show that $n$ must be equal to one and complete our proof by establishing the following lemma.

Lemma C. If B denotes the point set consisting of the sum of the boundaries of the complementary domains of a continuous curve $M$, and $P$ is a point of $\bar{B}-B$, then $P$ may be $\epsilon$-separated in $M$ by a single arc, for any positive number $\epsilon$.

There exists a simple closed curve $J_{1}$ which belongs to $M$, is of diameter less than $\epsilon$ and contains $P$ in its interior. As $P$ belongs to $\bar{B}-B$ there exists a complementary domain $D$ of $M$ in the interior of $J_{1}$ and a positive number $\epsilon^{\prime}$ such that no point of $J_{1}+D$ is within a distance $\epsilon^{\prime}$ of $P$. There exists a simple closed curve $J_{2}$ which belongs to $M$, is of diameter less than $\epsilon^{\prime}$, and contains $P$ in its interior. ${ }^{*}$ Then $J_{2}$ lies in the interior of $J_{1}$ and $D$ lies between $J_{1}$ and $J_{2}$. Now let $J_{3}$ be any simple closed curve which lies between $J_{1}$ and $J_{2}$ and contains a point of $D$. Let $G_{1}, G_{2}, G_{3}, \cdots$ be the set of all components of $J_{3} \cdot\left(E_{2}-M\right)$. This set contains at least one component and is countable. Each component $G_{i}$ lies in a complementary domain $D_{i}$ of $M$. Let $B_{i}$ be the boundary of $D_{i}$ and let

$$
G=\left(J_{3}-\sum G_{i}\right)+\sum B_{i} .
$$

* G. T. Whyburn, Concerning continua in the plane, loc. cit. 
Now (1) $G$ lies entirely between $J_{1}$ and $J_{2}$ except for points on $J_{1}$ and $J_{2}$, (2) $M$ contains $G$, (3) $G$ is a continuous curve, (4) $P$ lies in a bounded complementary domain of $G{ }^{*}$ The outer boundary of this domain is a simple closed curve $J_{4} \dagger$ which belongs to $M$, contains $P$ in its interior, and has two points $X$ and $Y$ on the boundary of some complementary domain $D_{i}$. Let $X U Y$ be an arc from $X$ to $Y$ which lies in $D_{i}$ except for $X$ and $Y$. The point $P$ lies in the interior of $J_{4}$ and thus it lies in the interior of one of the two simple closed curves formed by the arc $X U Y$ plus one of the arcs of $J_{4}$ from $X$ to $Y$. Call this arc $X V Y$. Then $X V Y$ is the desired $\epsilon$-separating arc, for $P$ lies in the interior of the simple closed curve $X U Y+X V Y$ and this simple closed curve lies in the set consisting of $J_{1}$ plus its interior.

3. THEOREM 1. In order that every arc of a continuous curve $M$ be extendible in $M$ in both directions it is necessary and sufficient that $M$ contain no point which can be $\epsilon$-separated in $M$ by an acyclic continuous curve for every positive number $\epsilon$.

The condition is sufficient. Every "point" arc may be extended because every point of a continuous curve, except end points, is interior to an arc of $M$. But an end point may be $\epsilon$-separated by a point for every $\epsilon$, and there exists no such point by our condition. Now let $\alpha$ be any true arc of $M$ and let $P$ and $Q$ be the end points of $\alpha$. We shall show that $\alpha$ is extendible in $M$ in the direction $Q P$. Suppose this is not the case. Let us consider the components of $M-\alpha$. No one of these has $P$ as a limit point for then $\alpha$ would be extendible in $M$ in the direction $Q P$, contrary to our supposition. Let $\epsilon$ be any positive number. There exists a point $X$ of $\alpha$ so that the diameter of the subarc $X P$ of $\alpha$ is less than $\epsilon$, and there exists a point $Y(X \neq Y)$ of the subarc $X P$ so that

* For detailed proof of these and the following statements, see page 204 of my paper, On the separation of points of a continuous curve by arcs and simple closed curves, loc. cit.

$\dagger$ R. L. Moore, Concerning continuous curves in the plane, Mathematische Zeitschrift, vol. 15 (1922), pp. 254-260, Theorem 4. 
the diameter of the subarc $Y P$ of $\alpha$ is less than $\epsilon / 2$. Since only a finite number of the components of $M-\alpha$ are of diameter greater than any given positive number, ${ }^{*}$ there exists a point $Z(Z \neq Y)$ on the subarc $Y P$ of $\alpha$ so that (1) no component of $M-\alpha$ of diameter greater than $\epsilon / 2$ has a limit point on $Z P$ and (2) no component of $M-\alpha$ has a limit point on both $Z P$ and $X Q$. Then the subarc $X Z$ is an $\epsilon$-separating set of $P$ in $M$. But this is contrary to the hypothesis. Hence $\alpha$ is extendible in $M$ in the direction $Q P$, and similarly in the direction $P Q$.

The condition is necessary. Suppose $M$ contains a point $P$ which can be $\epsilon$-separated by an acyclic continuous curve for every $\epsilon$. We shall show that this leads to a contradiction by constructing in $M$ an arc which cannot be extended in $M$ in one direction. By Lemma B, $P$ may be $\epsilon$-separated in $M$ by an arc. There exists a sequence $J_{1}, J_{2}, J_{3}, \cdots$ of simple closed curves such that (1) the interior of $J_{i}$ contains $J_{i+1}$ and $P,(2)$ the diameter of $J_{i}$ approaches zero as $i$ increases indefinitely, (3) $J_{i} \cdot M=A_{i} B_{i}$ consists of a single arc. When $A_{i} B_{i}$ is not a point, then $A_{i}$ and $B_{i}$ are the end points of $A_{i} B_{i}$. In the order from $A_{i}$ to $B_{i}$ let $C_{i}$ and $D_{i}$ denote the first and last limit points of the component of $M-A_{i} B_{i}$ that contains $P$. Let $E_{i}$ and $F_{i}(i>1)$ denote the first and last limit points on $A_{i} B_{i}$ of that component of $M-A_{i} B_{i}$ that contains $A_{i-1} B_{i-1}$. Each of the subarcs $C_{i} D_{i}$ and $E_{i} F_{i}$ of $A_{i} B_{i}$ is a cutting of $M$. The component of $M-C_{i} D_{i}$ containing $P$ lies in the interior of $J_{i}$, and the component of $M-E_{i} F_{i}$ containing $P$ lies in the interior of $J_{i-1}$. There exists an arc $\alpha$ with end points $D_{1}$ and $D_{2}$ and lying except for $D_{1}$ entirely in the component of $M-A_{1} B_{1}$ that contains $P . \dagger$ In the order from $D_{1}$ to $D_{2}$ there is a first point $G_{1}$ of $A_{2} B_{2}$ on $\alpha$. Consider the two arcs $C_{3} D_{3}$ and $E_{3} F_{3}$. Either one end point of $E_{3} F_{3}$ is not an interior point of $C_{3} D_{3}$ or both end

* W. L. Ayres, Note on a theorem concerning continuous curves, Annals of Mathematics, vol. 28 (1927), pp. 501-2.

$\dagger$ R. L. Wilder, Concerning continuous curves, Fundamenta Mathematicae, vol. 7 (1925), p. 342 . 
points of $E_{3} F_{3}$ are interior points of $C_{3} D_{3}$. In the first case let $H_{1}$ be this end point. And in this case there is an end point $H_{2}$ of $C_{3} D_{3}$ so that the subarc $H_{1} H_{2}$ of $A_{3} B_{3}$ contains the entire arc $C_{3} D_{3}$. In the latter case let $H_{1}$ be either end point of $E_{3} F_{3}$ and let $H_{2}$ be that end point of $C_{3} D_{3}$ so that the subarc $H_{1} H_{2}$ of $A_{3} B_{3}$ contains the entire arc $E_{3} F_{3}$. In either case, then, we have a subarc $H_{1} H_{2}$ of $A_{3} B_{3}$ so that $H_{1} H_{2}$ contains either $C_{3} D_{3}$ or $E_{3} F_{3}$. There exists* an arc $G_{2} H_{1}$ of $M$ which lies, except for one end point $G_{2}$ on $J_{2}$ and one end point $H_{1}$ on $J_{3}$, entirely between $J_{2}$ and $J_{3}$. In general, the arc $A_{2 n+1} B_{2 n+1}(n=1,2,3, \cdots)$ contains an arc $H_{2 n-1} H_{2 n}$ which contains one of the two arcs $C_{2 n+1} D_{2 n+1}$ or $E_{2 n+1} F_{2 n+1}$, and is such that $H_{2 n-1}$ is either $E_{2 n+1}$ or $F_{2 n+1}$ and $H_{2 n}$ is either $C_{2 n+1}$ or $D_{2 n+1}$. There exist two $\operatorname{arcs} G_{2 n} H_{2 n-1}$ and $H_{2 n} G_{2 n+1}$ of $M$ such that $G_{2 n}$ is a point of $J_{2 n}, G_{2 n+1}$ is a point of $J_{2 n+2}$, and these two arcs lie except for their end points entirely between $J_{2 n}$ and $J_{2 n+1}$ and $J_{2 n+1}$ and $J_{2 n+2}$ respectively. Let $\beta=P+C_{1} D_{1}+\operatorname{subarc} D_{1} G_{1}$ of $\alpha+\sum_{n=1}^{\infty}\left(\operatorname{subarc} G_{2 n-1} G_{2 n}\right.$ of $\left.A_{2 n} B_{2 n}\right)$

$$
\left.+\sum_{i=1}^{\infty} H_{i+1} G_{i+1}+\sum_{n=1}^{\infty} \text { (subarc } H_{2 n-1} H_{2 n} \text { of } A_{2 n+1} B_{2 n+1}\right) \text {. }
$$

Evidently $\beta$ is an arc of $M$ from $C_{1}$ to $P$. And further we may show that this arc may not be extended in $M$ in the direction $C_{1} P$ for each of the arcs $H_{2 n-1} H_{2 n}$ contains a cutting of $M$ and these cuttings of $M$ are such that the diameter of the component of $M-H_{2 n-1} H_{2 n}$ containing $P$ approaches zero as $n$ increases. Hence any arc of $M$ with $P$ as an end point contains a point of infinitely many of the arcs $H_{2 n-1} H_{2 n}$. Therefore $\beta$ cannot be extended in $M$ in the direction $C_{1} P$.

4. THEOREM 2. In order that every arc of the continuous curve $M$ be extendible in $M$ in both directions it is necessary and sufficient that (1) the sum $B$ of the boundaries of all the complementary domains of $M$ be a closed point set, (2) every point of $B$ be either a cut point of $M$ or belong to the boundaries of at least two complementary domains of $M$.

* See R. L. Wilder's paper cited in the footnote on p. 855 . 
The conditions are necessary. Suppose $\bar{B}-B$ contains a point $P$. By Lemma C, $P$ may be $\epsilon$-separated in $M$ by an arc for every positive $\epsilon$. But by Theorem $1, M$ contains no such point $P$. Therefore $\bar{B}-B$ is vacuous and the first condition is necessary. Now suppose $P$ is any point of $B$. If $C$ is any circle with center $P$, then $P$ is accessible from at least one complementary domain of $M+C$ since $B$ contains $P$. If for every circle $C, P$ is accessible from just one complementary domain of $M+C$, then $P$ may be $\epsilon$-separated, for any $\epsilon>0$, by an arc, from Lemma $A$. But this is impossible by Theorem 1 . Hence there is some circle $C$ so that $P$ is accessible from at least two complementary domains $D_{1}$ and $D_{2}$ of $M+C$. If $D_{1}$ and $D_{2}$ belong to different complementary domains of $M$, we have the latter part of condition (2). And if $D_{1}$ and $D_{2}$ belong to the same complementary domain of $M$, it may be shown that $P$ is a cut point of $M$, which is the first part of condition (2).

The conditions are sufficient. Let $P$ be a point of $M$. If $P$ is not a point of $B$, it is not a point of $\bar{B}$ by condition (1) and there exists a circle with center $P$ and radius $r$ such that every point of the interior of the circle belongs to $M$. Then $P$ cannot be $\epsilon$-separated by an acyclic continuous curve for $\epsilon<r$. If $P$ belongs to the boundary of just one complementary domain of $M, P$ is a cut point of $M$ and for $\epsilon$ sufficiently small, no cut point may be $\epsilon$-separated in $M$ by a set consisting of just one component. And if $P$ belongs to the boundaries of two complementary domains of $M$, we may show that any $\epsilon$-separating set, for $\epsilon$ sufficiently small, must contain more than one component. Hence $M$ contains no point which can be $\epsilon$-separated in $M$, for every positive $\epsilon$, by an acyclic continuous curve. By Theorem 1, every arc of $M$ is extendible in $M$ in both directions.

5. Theorem 3. If every arc of the continuous curve $M$ is extendible in $M$ in both directions, every point of $M$ lies on the boundary of some complementary domain of $M(M \equiv B)$.

By Theorem 2, the set $B$, the sum of the boundaries of the 
complementary domains of $M$, is a closed point set. Now suppose there is a point $P$ of $M-B$. Every complementary domain of $M$ is a complementary domain of the point set $B$ and in addition there is at least one complementary domain of $B$, all of whose points belong to $M$. Let $D$ denote the complementary domain of $B$ containing $P$. We shall assume that $D$ is bounded for we may always produce this by an inversion. Let $B_{1}$ denote the outer boundary of $D .{ }^{*}$ Then $B_{1}$ is the common boundary of two domains, one the exterior of $B_{1}$ and the other a bounded domain containing $D . \dagger$ Then no point of $B_{1}$ disconnects $B_{1} \neq \ddagger$ Then by a theorem due to R. L. Moore, $\S$ only a countable number of the points of $B_{1}$ are cut points of $M$. Hence, by Theorem 2, uncountably many points of $B_{1}$ are accessible from two complementary domains of $M$. Each of these points is also a limit point of $D$. There are only a countable number of complementary domains of $M$ and thus only a countable number of pairs of complementary domains of $M$. And by a theorem due to G. T. Whyburn, $\uparrow$ at most two points can be accessible from each of the two domains of a pair and at the same time be limit points of $D$. Thus in all only a countable number of points of $B_{1}$ can be accessible from two complementary domains of $M$. Therefore our assumption that $M-B$ is non-vacuous is false.

The University of Vienna, and

The University of Michigan

* R. L. Moore, loc. cit., p. 256.

† Ibid., Theorem 3, p. 258.

$\ddagger$ R. L. Moore, Concerning the common boundary of two domains, Fundamenta Mathematicae, vol. 6 (1924), pp. 203-213, Theorem 2.

$\S$ Concerning the cut-points of continuous curves and of other closed and connected point-sets, Proceedings of the National Academy of Sciences, vol. 9 (1923), pp. 101-106, Theorem B*.

I Concerning plane closed point sets which are accessible from certain subsets of their complements, Proceedings of the National Academy of Sciences, vol. 14 (1928), pp. 657-666, Theorem 1 and lines 1-3 of page 659. 